\title{
Expression of a Tumor-Associated Antigen, RCAS1, in Canine Mammary Tumors
}

\author{
Yasuhiko OKAMURA ${ }^{1)}$, Tomoya HARAGUCHI ${ }^{1)}$, Masahiro MORIMOTO ${ }^{2)}$, Masaru OKUDA ${ }^{3)}$, Satoshi UNE $^{1)}$, \\ Munekazu NAKAICHI ${ }^{1)}$ and Yasuho TAURA ${ }^{1) *}$ \\ ${ }^{1)}$ Departments of Veterinary Surgery, ${ }^{2)}$ Veterinary Pathology and ${ }^{3)}$ Veterinary Internal Medicine, Faculty of Agriculture, Yamaguchi \\ University, Yamaguchi, 1677-1 Yoshida, Yamaguchi 753-8515, Japan
}

(Received 24 September 2003/Accepted 19 January 2004)

ABSTRACT. Receptor-binding cancer antigen expressed on SiSo cells (RCAS1), one of novel cancer cell-surface antigens, is strongly expressed in invasive cancers. RCAS1 inhibits the in vitro growth of lymphocytes such as T cells and natural killer (NK) cells, and induces apoptotic cell death. We investigated the expression of RCAS1 in canine mammary tumor cell lines and tumor cells by immunohistochemistry, and also in situ deoxyribonucleic acid (DNA) fragmentation in tumor-infiltrating lymphocytes (TILs) by the terminal deoxynucleotidyl transferase mediated deoxyuridine triphosphate nick end labeling (TUNEL) method. All canine mammary tumor cell lines expressed RCAS1 at both the messenger ribonucleic acid (mRNA) and protein level. Immunohistochemically, RCAS1 was negative in $100 \%$ of normal mammary glands, but was expressed in $100 \%$ of malignant tumors examined. In most malignant mammary tumors, RCAS1 was localized in the cytoplasm with no polarity of expression. In benign mammary tumors, it was detected on the luminal surface of the tumor cell. RCAS1 expression or localization was significantly correlated with malignancy. In situ DNA fragmentation of CD3-positive TILs was observed in RCAS1-expressing tumors. RCAS1-expressing tumors, indicating a possible induction of apoptotic cell death in TILs through RCAS1 expression. These observations suggest that RCAS1 probably plays an important role in tumor progression and escape from immune surveillance in canine mammary tumors.

KEY WORDS: canine mammary tumor, DNA fragmentation, immunohistochemistry, receptor-binding cancer antigen expressed on SiSo cells (RCAS1), tumor infiltrating lymphocyte (TILs).

J. Vet. Med. Sci. 66(6): 651-658, 2004

Mammary neoplasms are among the most common tumors in intact female dogs $[18,27]$. The median age of tumor manifestation is between 10 and 11 years old, with rare occurrence in dogs younger than 4 years. On histological examination, approximately $50 \%$ of canine mammary tumors are considered malignant, and the most common tumors are solid carcinomas and adenocarcinomas. However, because of the heterogeneity of histopathological types and clinical presentation of canine mammary tumors, histological evidence of malignancy does not imply a malignant clinical course. In human breast cancer there are many biochemical markers used for detection and management of patients [4]. These markers can help in selecting subjects at high risk of developing the inherited form of the disease (BRCA1 and BRCA2), in assessing prognosis (uPA and PAI-1), and in guiding therapy and monitoring patients with diagnosed disease (CA15-3 and BR27.29). The appropriate use of these markers will lead to better management of patients with breast cancer. In contrast, in veterinary practice tumor markers are not commonly used, and there is a necessity to develop new strategies for the diagnosis and prognosis of canine mammary tumors.

Receptor-binding cancer antigen expressed on SiSo cells (RCAS1) was recognized by the mouse monoclonal antibody 22-1-1 against the human uterine adenocarcinoma cell line SiSo [28]. RCAS1 is identical to the estrogen receptor-binding fragment-associated gene 9 (EBAG9).

\footnotetext{
* Correspondence to: Taura, Y., Department of Veterinary Surgery, Faculty of Agriculture, Yamaguchi University, 1677-1 Yoshida, Yamaguchi 753-8515, Japan.
}

EBAG9 has been identified as an estrogen-responsive gene from a complementary deoxyribonucleic acid (cDNA) library of MCF7 human breast cancer cells [39]. RCAS1 consists of 213 amino acids and is deduced as a type II membrane protein that forms homo-oligomers through its C-terminal coiled-coil structures [20]. RCAS1 is highly conserved among humans, mice and dogs [20, 23, 34]. Canine RCAS1 cDNA shows high homology with its human $(91.7 \%)$ and mouse $(89.9 \%)$ counterparts [23].

Immunohistochemical analysis has revealed RCAS1 to be expressed in both human uterine and ovarian cancers [25, 28-31]. RCAS1 has also been detected in nongynecological cancers such as human breast carcinomas [26, 32, 36], esophageal squamous cell carcinomas [19], gastric adenocarcinomas $[6,16]$, hepatocellular carcinomas [2, 21], lung cancers $[13,14,22]$, and pancreatic adenocarcinomas [1, 19]. Expression of RCAS1 is associated with a poor prognosis in these cancer patients. Moreover, RCAS1 acts as a ligand for a putative receptor present on normal peripheral lymphocytes such as T, B, and natural killer (NK) cells [20]. RCAS1 inhibits proliferation of receptor positive cells in vitro and induces apoptotic cell death, suggesting that tumor cells can evade immune surveillance by increased expression of RCAS1, which would act to suppress clonal expansion and induce apoptosis in immune cells possessing RCAS1 receptors. Very little is known about RCAS1 protein expression in canine mammary tumors. Therefore, we investigated the relationship between RCAS 1 expression and clinicopathological variables of canine mammary tumors by immunohistochemical analysis. 


\section{MATERIALS AND METHODS}

Cell lines: The human breast cancer cell line as a positive control, MCF7 (Cell Resource Center for Biomedical Research, Institute of Development, Aging and Cancer, Tohoku University, Sendai, Japan) and the canine mammary tumor cell lines CIP-p and CIP-m (Department of Veterinary Surgery, Graduate School of Agricultural and Life Sciences, The University of Tokyo, Tokyo, Japan) were maintained in RPMI-1640 (Gibco BRL, St. Louis, MO, U.S.A.) supplemented with $10 \%$ fetal bovine serum (FBS, Vitromex, Vilshofen, Germany).

Tissue samples: Normal mammary gland tissues were obtained from three dogs with no tumors. Mammary tumor specimens were obtained from dogs undergoing surgical resection at the Yamaguchi University Veterinary Hospital between July 2001 and July 2003. The animals were aged from 5 to 14 years. The histological classification was based on the World Health Organization (WHO) criteria [17]. Tissue samples had been diagnosed microscopically and included 4 benign mixed tumors, one simple adenoma, two complex adenoma, one osteosarcoma, 9 complex carcinomas; and 5 simple carcinomas, including three tubulopapillary carcinomas, one solid carcinoma, and one anaplastic carcinoma. The clinical staging was classified according to the WHO clinical stage classification [24, 27].

Reverse transcription - polymerase chain reaction ( $R T$ $P C R)$ : RCAS1 expression in the canine mammary tumor cell lines, CIP-p and CIP-m, were examined by RT-PCR. Total ribonucleic acid (RNA) was extracted from the cell lines with a QIAamp ${ }^{\circledR}$ RNA Blood Mini Kit (Quiagen, Hilden, Germany) according to the instructions of the manufacturer. Reverse transcription of the RNA was performed with a Sensiscript ${ }^{\mathrm{TM}}$ RT kit (Quiagen) using a 38 bases oligonucleotide primer (AP) with $17 \mathrm{dT}$ bases, followed by an adaptor sequence, 5'-GGCCACGCGTCGACTAC-3'. Takara EX Taq ${ }^{\mathrm{TM}}$ (hot start version, Takara, Otsu, Shiga, Japan) was used in PCR for amplification of the canine RCAS1 cDNA. The PCR conditions were as follows: 28 cycles at $94^{\circ} \mathrm{C}$ for $30 \mathrm{sec}, 60^{\circ} \mathrm{C}$ for $30 \mathrm{sec}$, and $72^{\circ} \mathrm{C}$ for 1 min. The PCR products were electrophoresed in $2 \%$ agarose gels. The sets of primers for canine RCAS1 consisted of a forward primer of 5'-AGAGGACGGAAATTAAGTGGAGACCA-3' and a reverse primer of 5'-GTTGGTGTCATGTCCTTAAAATAGT-3, [36]. Canine Glyceraldehyde-3-phosphate dehydrogenase (forward, 5' GCCAAAAGGGTCATCATCTC-3'; reverse, 5'-GGCCATCCACAGTCTTCT-3') was amplified as an internal control [7].

Immunocytochemistry: MCF7, CIP-p and CIP-m were cultivated on chamber slide (Asahi Techno Glass Co., Tokyo, Japan). Endogenous peroxidase was blocked with $1.5 \%$ hydrogen peroxide in methanol for $10 \mathrm{~min}$. To block nonspecific binding, cancer cell lines were incubated for 30 min with $3 \%$ bovine serum albumin. Cells were incubated with primary antibody (22-1-1, Medical \& Biological Lab.,
Nagoya, Japan) in a humid chamber at $4^{\circ} \mathrm{C}$ overnight. Negative controls were obtained by replacing the primary antibody by normal mouse IgM (Dako, Carpinteria, CA, U.S.A.). Cell lines were followed by several washes and an hour incubation with anti-mouse EnVision+ (Dako) at room temperature. The antigen-antibody complex was visualized with 3,3'-diaminobenzidine (DAB) solution (Dako). Sections were counterstained with hematoxylin (Dako).

Immunohistochemistry: For immunohistochemical analysis, the dextran polymer methodology (EnVision+ kit; Dako) was used for frozen tissue specimens embedded in Tissue-Tek ${ }^{\circledR}$ Optimal Cutting Temperature compound (Sakura Finetechnical Co., Ltd., Tokyo). Briefly, tissue sections $\left(6 \mu \mathrm{m}\right.$ thick) were fixed with acetone at $-20^{\circ} \mathrm{C}$ for 10 min. Endogenous peroxidase was blocked with $1.5 \%$ hydrogen peroxide in methanol for $10 \mathrm{~min}$. The sections were washed three times with TBS-T (Tris buffer saline containing $0.1 \%$ Tween 20 ), and then incubated with $3 \%$ bovine serum albumin for 30 min to block nonspecific antibody binding sites. Sections were then incubated with primary antibody (22-1-1) in a humid chamber at $4{ }^{\circ} \mathrm{C}$ overnight. After being rinsed in TBS-T, sections were incubated with anti-mouse EnVision+ (Dako) for $1 \mathrm{hr}$ at room temperature. The antigen-antibody complex was visualized with DAB solution (Dako). Sections were counterstained with hematoxylin (Dako). Negative controls were treated in the same way, but the primary antibody was replaced by normal mouse IgM (Dako). RCAS1 immunoreactivity was classified as negative (-, less than $5 \%$ of cell stained), 1+ $(5 \% \leq$ cell stain $<25 \%), 2+(25 \% \leq$ cell stain $<50 \%)$ and $3+$ (more than $50 \%$ cell stained) [15].

In situ detection of DNA fragmentation: In situ detection of DNA fragmentation was achieved by terminal deoxynucleotidyl transferase (TdT) mediated deoxyuridine triphosphate (dUTP) nick end labeling (TUNEL) procedure, using an in situ apoptosis detection kit (Takara) with some modification to the manufacturer's instructions. Briefly, the TUNEL method is a tailing reaction in which residues of fluorescein isothiocyanate -labeled dUTP are added to the 3'-OH end of DNA by TdT. For double immunofluorescent labeling, tumor-infiltrating lymphocytes (TILs) were examined by using mouse anti-canine CD3 antibody $(1: 100$, Serotec, Oxford, U.K.). Anti-mouse IgG TRITC conjugate (1:200, Sigma, Steinheim, Germany) was used as a fluorescent secondary antibody. Nuclei were labeled with Hoechst 33258 (Wako Pure Chemicals Industries, Ltd., Osaka, Japan). Slides were viewed at $400 \times$ under a fluorescent microscope (BHS-RFK, Olympus, Tokyo). Acquisition and processing of double immunofluorescent images of the canine mammary tumor sections were performed with the Photoshop computer program (Adobe Sys. Inc.; San Jose, CA).

Statistical analysis: The relationship between RCAS1 expression and clinicopathological variables was investigated by Fisher's exact test. Differences were considered significant when $P$-values were less than 0.05 . 


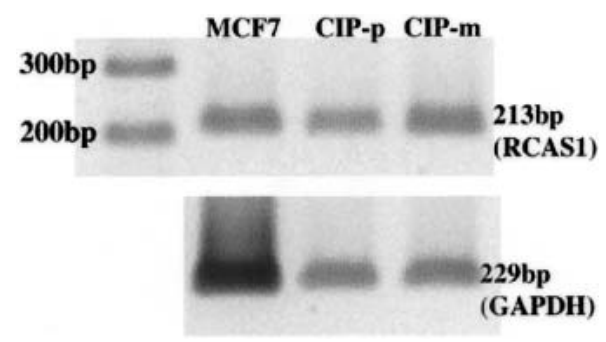

Fig. 1. Expression of Receptor-binding cancer antigen expressed on SiSo cells (RCAS1) messenger ribonucleic acid (mRNA) in canine mammary tumor cell lines (CIP-p and CIP-m) was analyzed by Reverse transcription - polymerase chain reaction (RT-PCR). For positive control, human breast cancer cell line (MCF7) was used. Canine Glyceraldehyde-3-phosphate dehydrogenase (GAPDH) was amplified as an internal control.

\section{RESULTS}

RCAS1 expression in canine mammary tumor cell lines: To ascertain whether canine mammary tumors expressed RCAS1, we used RT-PCR and immunocytochemical analysis for examine the canine mammary tumor cell lines CIP-p and CIP-m. We used the human breast cancer cell line MCF7 as a positive control. First, RCAS1 messenger RNA (mRNA) expression was analyzed by RT-PCR; mRNA was produced in all canine mammary tumor cell lines tested (Fig. 1). Next, to investigate RCAS1 protein expression in MCF7, CIP-p and CIP-m cell lines, cells were subjected to

A

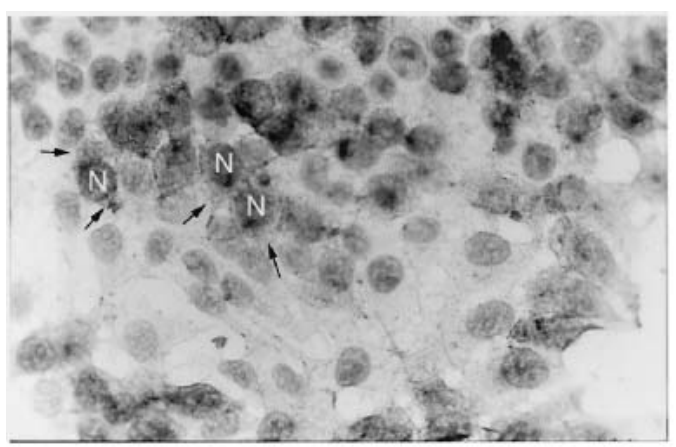

C

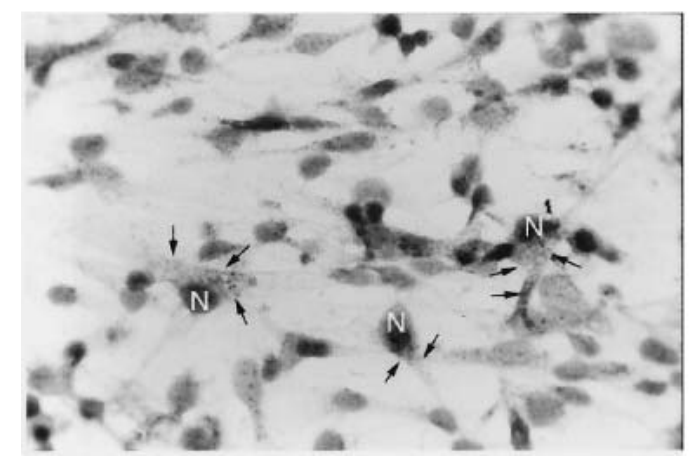

immunocytochemical analysis. RCAS1 protein was expressed in CIP-p and CIP-m cytoplasm (Fig, 2). In particular, CIP-m showed a stronger expression of RCAS1 proteins in the cytoplasm.

Immunohistochemical analysis of RCAS1 expression: RCAS1 expression was examined immunocytochemically in frozen tissue specimens. Negative controls showed no immunoreactivity. RCAS1 expression was negative in $100 \%$ of normal mammary gland and myoepithelial cells (Figs. 3A, 4A). Four of the 7 benign tumors were classified as belonging to the low group (Figs. 3B, 4B). RCAS1 was positive in $100 \%$ of specimens of complex carcinoma, simple carcinoma and osteosarcoma (Table 1). RCAS1 was found in the cytoplasm and on the membrane of cancer cells. RCAS1 expression patterns in representative canine mammary tumor specimens are shown in Fig. 4. There was no correlation between RCAS1 expression and clinicopathological variables (age, primary tumor size, regional lymph node status, distant metastasis, and stage grouping). In contrast, RCAS 1 expression and tumor malignancy were correlated significantly (Table 2). Interestingly, tumors exhibited different staining intensities for RCAS1. Expression of RCAS1 in the cytoplasm without polarity was defined as a 'WP' pattern (Figs. 3D, 4D). Expression of RCAS1 on the luminal surface of the tumor cells was defined as a 'LS' pattern (Figs. 3B, 4B). In $100 \%$ (8 of 8 cases) of stage III to V canine mammary tumors, RCAS1 existed only in the WP pattern. Overall, $93.3 \%$ (14 of 15 cases) of malignant mammary tumors showed the WP pattern. In contrast, $85.7 \%$ (6 of 7 cases) of benign mammary tumors showed the LS pattern. There was a significant correlation between RCAS1 localization pattern and primary tumor size, stage grouping

B

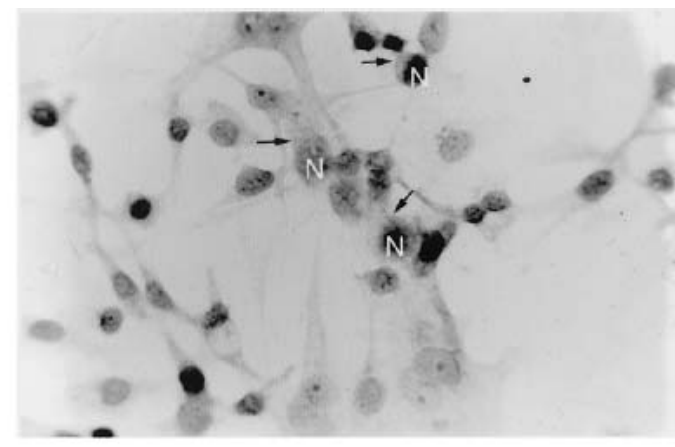

Fig. 2. RCAS1 is revealed in canine mammary tumor cell lines by immunocytochemistry. (A) MCF7 cells express the cytoplasmic level of RCAS1; (B) CIP-p cells express the cytoplasmic level of RCAS1; (C) showing a stronger cytoplasmic level of RCAS1 in CIP-m. Partially, $\mathrm{N}$ indicates nuclei and arrows indicate RCAS1 expression in cytoplasm. Magnification is $400 \times$. 
A

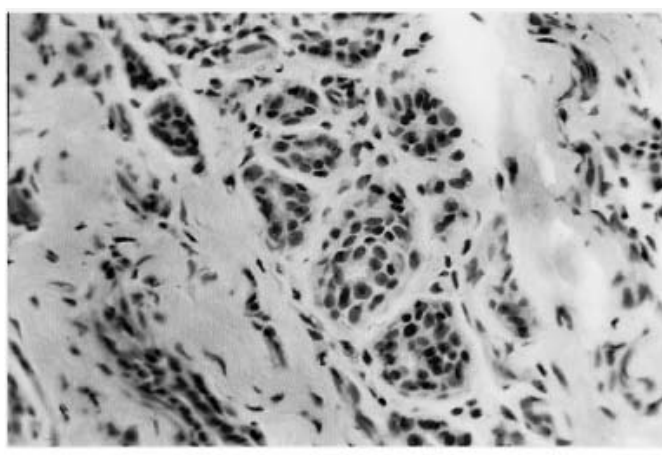

$\mathbf{C}$

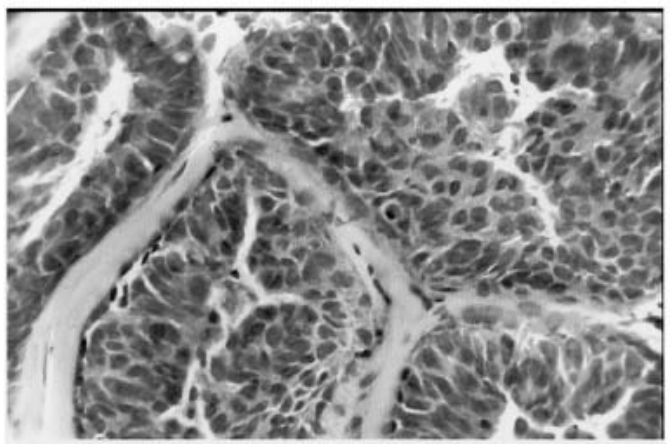

B

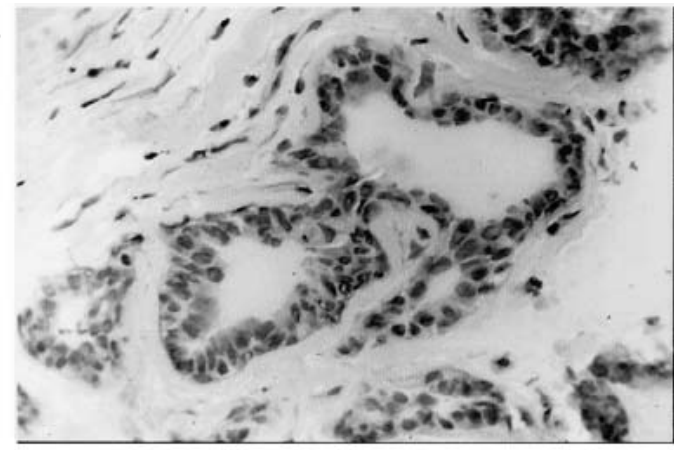

D

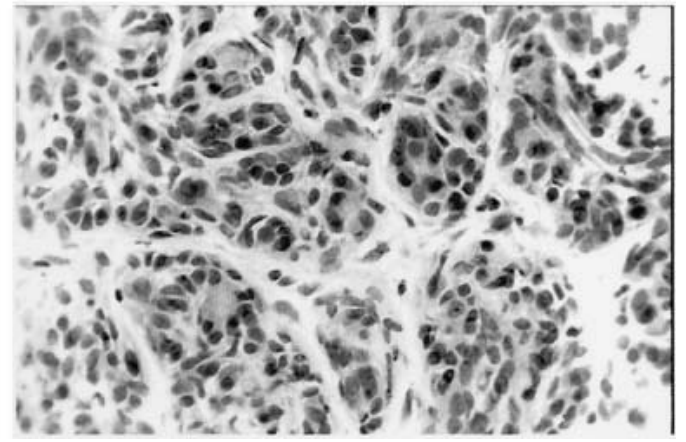

Fig. 3. Tissue sections were stained with hematoxylin and eosin. Tissue sections in the following cases: normal mammary gland (A), benign mixed tumor (B), simple carcinoma (tubulopapillary carcinoma, C), complex carcinoma (D). Magnification is $400 \times$.

Table 1. Immunodetection data

\begin{tabular}{lccccc}
\hline & & \multicolumn{2}{l}{ Low group } & \multicolumn{2}{c}{ High group } \\
\cline { 2 - 6 } Histologic classification & Number of cases & - & + & $2+$ & $3+$ \\
\hline Normal mammary gland & 3 & 3 & 0 & 0 & 0 \\
\hline Benign mixed tumor & 4 & 0 & 3 & 1 & 0 \\
\hline Simple adenoma & 1 & 1 & 0 & 0 & 0 \\
\hline Complex adenoma & 2 & 0 & 0 & 2 & 0 \\
\hline Complex carcinoma & 9 & 0 & 0 & 3 & 6 \\
\hline Simple carcinoma & 5 & 0 & 0 & 2 & 3 \\
\hline \multicolumn{1}{c}{ Tubulopapillary carcinoma } & $(3)$ & $(0)$ & $(0)$ & $(1)$ & $(2)$ \\
\hline Solid carcinoma & $(1)$ & $(0)$ & $(0)$ & $(1)$ & $(0)$ \\
\hline Anaplastic carcinoma & $(1)$ & $(0)$ & $(0)$ & $(0)$ & $(1)$ \\
\hline Osteosarcoma & 1 & 0 & 0 & 0 & 1 \\
\hline Bencyyyyyy
\end{tabular}

Benign mixed tumor, simple adenoma and complex adenoma are classified as benign tumor. Complex carcinoma, simple carcinoma (tubulopapillary, solid and anaplastic carcinoma) and osteosarcoma are classified as malignant tumor. Receptor-binding cancer antigen expressed on SiSo cells (RCAS1) immunoreactivity was classified as negative (-, less than $5 \%$ of cell stained), $1+(5 \% \leq$ cell stain $<25 \%), 2+(25 \% \leq$ cell stain $<50 \%)$ and $3+$ (more than $50 \%$ cell stained).

and malignancy of the tumor (Table 3).

In situ detection of DNA fragmentation: We tried to detect DNA-fragmentation in lymphocytes around the RCAS1-positive canine mammary tumors by the TUNEL method. DNA fragmentation was observed in CD3-positive
TILs (Fig. 5). All RCAS1 positive tumors (21 samples) were observed in DNA fragmented CD3+ TILs. We were unable to detect DNA fragmentation of CD3+ TILs in normal mammary tissues ( 3 samples) and a RCAS1 negative sample. 
Table 2. Association between RCAS1 expression and clinicopathological variables

\begin{tabular}{|c|c|c|c|c|}
\hline \multirow[b]{2}{*}{ Clinicopathological variables } & \multirow[b]{2}{*}{ Number of cases } & \multicolumn{3}{|c|}{ Degree of RCAS1 expression } \\
\hline & &,$- 1+$ & $2+, 3+$ & $P$ value \\
\hline \multicolumn{5}{|l|}{ Age } \\
\hline$<10$ & 9 & 3 & 6 & \multirow{2}{*}{0.2643} \\
\hline$\geq 10$ & 13 & 1 & 12 & \\
\hline \multicolumn{5}{|l|}{ T: Primary tumor } \\
\hline $\mathrm{T} 1$ & 10 & 3 & 7 & \multirow{2}{*}{0.2932} \\
\hline $\mathrm{T} 2, \mathrm{~T} 3$ & 12 & 1 & 11 & \\
\hline \multicolumn{5}{|l|}{$\mathrm{N}$ : Regional lymph node status } \\
\hline No & 16 & 4 & 12 & \multirow{2}{*}{0.5407} \\
\hline $\mathrm{N} 1, \mathrm{~N} 2$ & 6 & 0 & 6 & \\
\hline \multicolumn{5}{|l|}{ M: Distant metastasis } \\
\hline M0 & 18 & 4 & 14 & \multirow{2}{*}{0.5538} \\
\hline M1 & 4 & 0 & 4 & \\
\hline \multicolumn{5}{|l|}{ Stage grouping } \\
\hline Stage I or II & 14 & 3 & 11 & \multirow{2}{*}{0.2727} \\
\hline Stage III or IV or V & 8 & 0 & 8 & \\
\hline \multicolumn{5}{|l|}{ Histological type } \\
\hline Benign tumor & 7 & 4 & 3 & \multirow{2}{*}{0.0227} \\
\hline Marignant tumor & 15 & 0 & 15 & \\
\hline
\end{tabular}

The clinical staging was classified according to the World Health Organization clinical stage classification. $P$ values less than 0.05 were considered as significant.

Table 3. Association between RCAS1 localization pattern and clinicopathological variables

\begin{tabular}{|c|c|c|c|c|}
\hline \multirow[b]{2}{*}{ Clinicopathological variables } & \multirow[b]{2}{*}{ Number of cases } & \multicolumn{3}{|c|}{ RCAS1 localization pattern } \\
\hline & & LS pattern & WP pattern & $P$ value \\
\hline \multicolumn{5}{|l|}{ Age } \\
\hline$<10$ & 9 & 5 & 4 & \multirow{2}{*}{0.0743} \\
\hline$\geq 10$ & 13 & 2 & 11 & \\
\hline \multicolumn{5}{|l|}{ T: Primary tumor } \\
\hline $\mathrm{T} 1$ & 10 & 6 & 4 & \multirow{2}{*}{0.0201} \\
\hline $\mathrm{T} 2, \mathrm{~T} 3$ & 12 & 1 & 11 & \\
\hline \multicolumn{5}{|l|}{$\mathrm{N}$ : Regional lymph node status } \\
\hline No & 15 & 6 & 9 & \multirow{2}{*}{0.1206} \\
\hline $\mathrm{N} 1, \mathrm{~N} 2$ & 7 & 0 & 7 & \\
\hline \multicolumn{5}{|l|}{ M: Distant metastasis } \\
\hline M0 & 18 & 7 & 11 & \multirow{2}{*}{0.2632} \\
\hline M1 & 4 & 0 & 4 & \\
\hline \multicolumn{5}{|l|}{ Stage grouping } \\
\hline Stage I or II & 14 & 7 & 7 & \multirow{2}{*}{0.0225} \\
\hline Stage III or IV or V & 8 & 0 & 8 & \\
\hline \multicolumn{5}{|l|}{ Histological type } \\
\hline Benign tumor & 7 & 6 & 1 & \multirow[t]{2}{*}{0.0006} \\
\hline Marignant tumor & 15 & 1 & 14 & \\
\hline
\end{tabular}

Expression of RCAS1 in the cytoplasm without polarity was defined as a 'WP' pattern, and on the luminal surface of the tumor cells was defined as a 'LS' pattern. $P$ values less than 0.05 were considered as significant.

\section{DISCUSSION}

We investigated the expression of RCAS1 in canine mammary tumors; very little is known about RCAS1 involvement in canine cancers. Canine mammary tumor cell lines (CIP-p and CIP-m) expressed RCAS1 at not only the mRNA level but also the protein level. The human RCAS1 gene is located at chromosome 8q23 [12]. The copy number of $8 \mathrm{q} 23$ is frequently increased in some cancers, such as breast, renal, and esophageal $[33,35,38,40]$. Furthermore, gains of $8 \mathrm{q} 23$ are involved in lymph node metastasis [33]. Therefore, we expected that canine RCAS1 

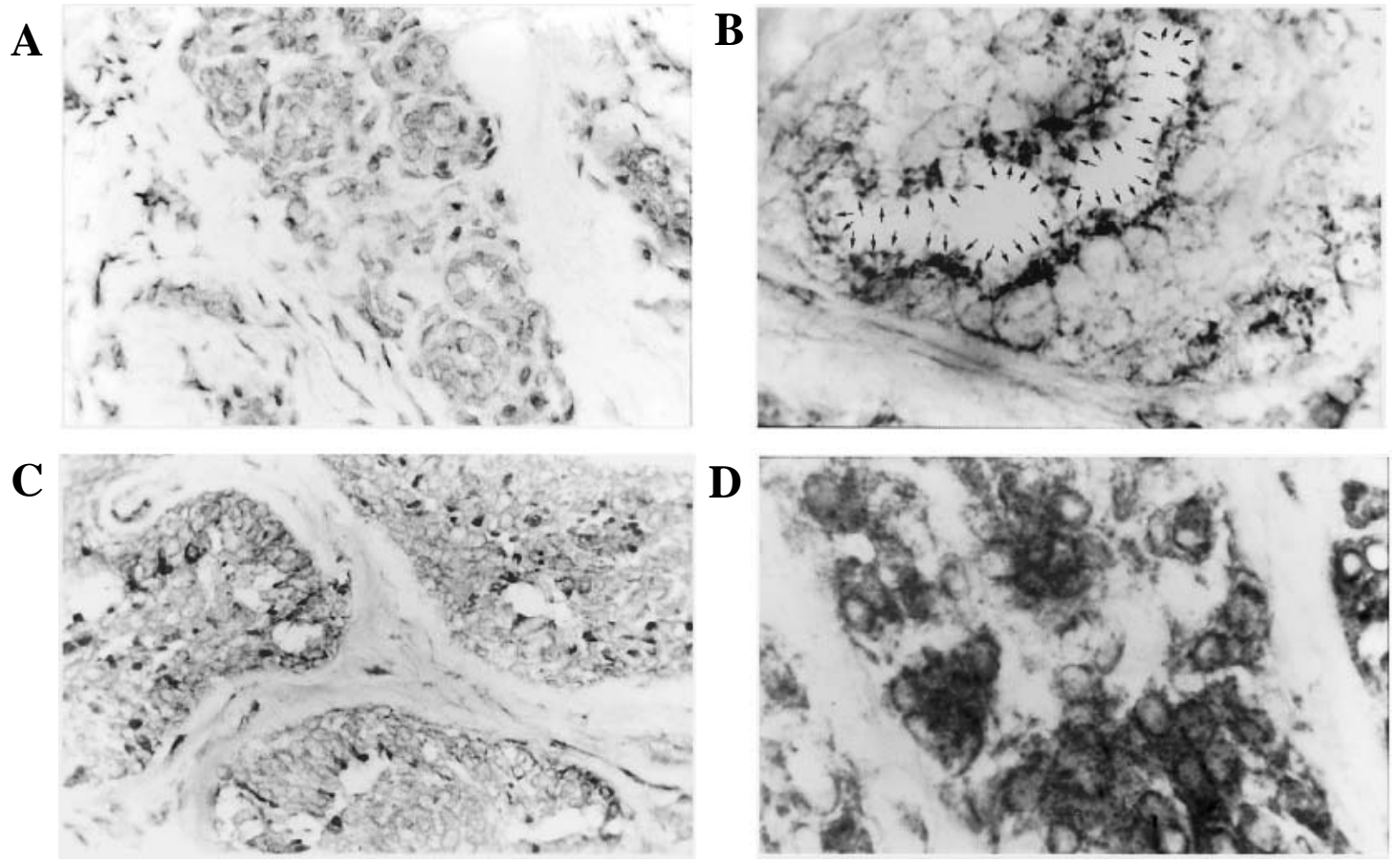

Fig. 4. Tissue sections were stained with 22-1-1 monoclonal antibody (MoAb). (A) Normal mammary gland and myoepithelial cells were not stained by $22-1-1$ MoAb. RCAS1 immunoreactivity was classified as (B) $1+(5 \% \leq$ cell stain $<25 \%$, benign mixed tumor), (C) $2+(25 \% \leq$ cell stain $<50 \%$, tubulopapillary carcinoma) and (D) $3+$ (more than $50 \%$ cell stained, complex carcinoma). (B) RCAS1 found in the luminal surface of the tumor cells (LS pattern). Arrows indicate. (C, D) RCAS1 found in the cytoplasm without polarity (WP pattern). (Magnification (A, C) $400 \times,(B, D) 1,000 \times$ )

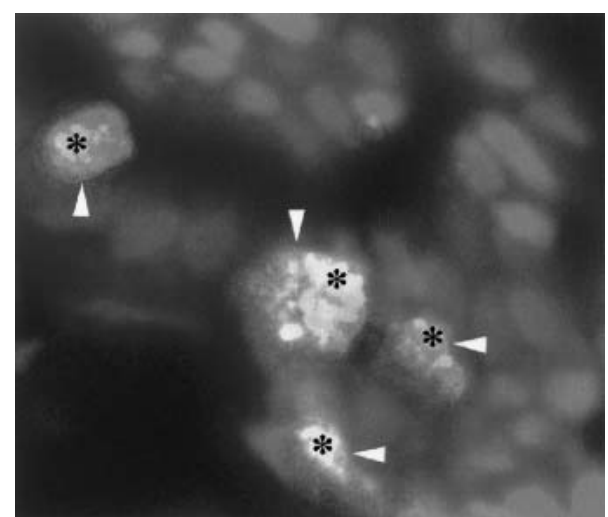

Fig. 5. Deoxyribonucleic acid (DNA) fragmentation of tumor-infiltrating lymphocytes (TILs) was detected in a case of complex carcinoma. Arrows indicate CD3+ TILs. Asterisks indicate fragmented DNA. Others are Hoechst 33258 labeled nuclei. Magnification is $400 \times$.

expression would be correlated with carcinogenesis of the mammary gland. In many kinds of human cancer, a high level of expression of RCAS1 is significantly correlated with tumor progression and poor prognosis $[6,9,13-15,19$, $22,31]$. In our study, $100 \%$ of marignant tumors were classified as belonging to the high expression group. Normal mammary tissues (100\%) and benign mixed tumors (57.1\%) were classified in the low expression group (Table 1). Our results showed that the level of RCAS1 expression was significantly related to histological classification, suggesting that RCAS1 expression is correlated with the degree of malignancy of the canine mammary tumor. We defined two RCAS1 localization patterns, the WP and LS patterns. RCAS1 localization patterns were significantly correlated with primary tumor size, stage grouping and malignancy. Differences in RCAS1 localization patterns have been observed in human breast cancer, hepatocellular carcinoma and gastric cancer $[2,16,26]$. Similar findings have also been reported for some cancer-associated antigens, including carcinoembrionic antigen (CEA) and carbohydrate antigen 19-9 (CA19-9) [8, 11]. The "loss of polar distribution" of membrane-associated antigens in cells causes malignant transformation. Therefore, the localization of RCAS1 will be correlated with the degree of malignancy of canine mammary tumors.

We were able to detect DNA fragmentation of CD3+ TILs in canine mammary tumors (Fig. 4). RCAS1 inhibited the in vitro growth of its receptor-expressing cells and induced their apoptotic cell death [20]. Putative RCAS1 receptors are expressed on peripheral blood lymphocytes (PBLs) such as T cells and NK cells. Recombinant and soluble RCAS1 inhibit cell proliferation and induce DNA fragmentation of PBLs. Moreover, fragmentation of the DNA 
in TILs has been observed in RCAS1-expressing human tumors (biliary adenocarcinoma, lung cancer, and gastric cancer) $[5,6,13]$. The majority of TILs in human tumors are $\mathrm{T}$ cells, suggesting an activation of the host defense mechanism [37]. In the veterinary sphere, it is reported that TILs also existed in canine transmissible venereal sarcomas (CTVS), where they are associated with tumor regression, not progression $[3,10,25]$. Therefore, RCAS1 probably induces apoptotic cell death of its receptor-positive TILs and contributes to the escape of the tumor from the control of the immune system. Furthermore, it is necessary to collect more samples and examine correlation between the degree of RCAS1 expression and apoptotic cell death of TILs in canine mammary tumors. Moreover, we should examine the expression of RCAS 1 in another kinds of canine tumor.

Soluble RCAS1 is present in the culture media of breast cancer and cholangiocarcinoma cell lines, the vaginal discharges of uterine cervical cancer patients, and the sera of pancreatic cancer patients $[1,5,26,28]$. The determination of serum RCAS1 expression by ELISA is now useful in pancreatic cancer [1]. Further studies are needed to clarify whether soluble RCAS1 is present in canine mammary tumors and whether serum RCAS1 is a useful marker in this disease.

In conclusion, we demonstrated that canine mammary tumor cells express RCAS1 protein. RCAS1 protein expression and expression localization were correlated significantly with the degree of tumor malignancy. Moreover, our results suggest that RCAS1 protein on canine mammary tumor cells plays a role in helping the cells to escape from immune surveillance. The relationship between RCAS1 expression and prognosis in canine mammary tumors is unclear but warrants examination. RCAS1 could be useful as a new marker in screening procedures for canine mammary tumors.

ACKNOWLEDGEMENT. We thank Dr. Nobuo Sasaki (Department of Veterinary Surgery, Graduate School of Agricultural and Life Sciences, The University of Tokyo, Tokyo, Japan) for providing the canine mammary cell lines (CIP-p and CIP-m).

\section{REFERENCES}

1. Akashi, T., Oimomi, H., Nishiyama, K., Nakashima, M., Arita, Y., Sumii, T., Kimura, T., Ito, T., Nawata, H. and Watanabe, T. 2003. Expression and diagnostic evaluation of the human tumor-associated antigen RCAS1 in pancreatic cancer. Pancreas 26: $49-55$.

2. Aoki, T., Inoue, S., Imamura, H., Fukushima, J., Takahashi, S., Urano, T., Hasegawa, K., Ogushi, T., Ouchi, Y. and Makuuchi, M. 2003. EBAG9/RCAS1 expression in hepatocellular carcinoma: correlation with tumour dedifferentiation and proliferation. Eur. J. Cancer 39: 1552-1561.

3. Barber, M. R. and Yang, T. J. 1999. Tumor infiltrating lymphocytes: CD8+ lymphocytes in canine transmissible venereal sarcomas at different stages of tumor growth. Anticancer Res.
19: $1137-1142$.

4. Duffy, M. J. 2001. Biochemical markers in breast cancer: which ones are clinically useful? Clin. Biochem. 34: 347-352.

5. Enjoji, M., Nakashima, M., Nishi, H., Choi, I., Oimomi, H., Sugimoto, R., Kotoh, K., Taguchi, K., Nakamuta, M., Nawata, H. and Watanabe, T. 2002. The tumor-associated antigen, RCAS1, can be expressed in immune-mediated diseases as well as in carcinomas of biliary tract. J. Hepatol. 36: 786-792.

6. Fukuda, K., Tsujitani, S., Maeta, Y., Yamaguchi, K., Ikeguchi, M. and Kaibara, N. 2002. The expression of RCAS1 and tumor infiltrating lymphocytes in patients with $\mathrm{T} 3$ gastric carcinoma. Gastric Cancer 5: 220-227.

7. Grone, A., Weckmann, M. T., Capen, C. C. and Rosol, T. J. 1996. Canine glyceraldehyde-3-phosphate dehydrogenase complementary DNA: polymerase chain reaction amplification, cloning, partial sequence analysis, and use as loading control in ribonuclease protection assays. Am. J. Vet. Res. 57: 254257.

8. Hamada, Y., Yamamura, M., Hioki, K., Yamamoto, M., Nagura, H. and Watanabe, K. 1985. Immunohistochemical study of carcinoembryonic antigen in patients with colorectal cancer. Correlation with plasma carcinoembryonic antigen levels. Cancer 55: 136-141.

9. Hiraoka, K., Hida, Y., Miyamoto, M., Oshikiri, T., Suzuoki, M., Nakakubo, Y., Shinohara, T., Itoh, T., Shichinohe, T., Kondo, S., Kasahara, N. and Katoh, H. 2002. High expression of tumor-associated antigen RCAS1 in pancreatic ductal adenocarcinoma is an unfavorable prognostic marker. Int. J. Cancer 99: 418-423.

10. Hsiao, Y. W., Liao, K. W., Hung, S. W. and Chu R.M. 2002. Effect of tumor infiltrating lymphocytes on the expression of MHC molecules in canine transmissible venereal tumor cells. Vet. Immunol. Immunopathol. 87: 19-27.

11. Ichihara, T., Nagura, H., Nakao, A., Sakamoto, J., Watanabe, T. and Takagi, H. 1988. Immunohistochemical localization of CA 19-9 and CEA in pancreatic carcinoma and associated diseases. Cancer 61: 324-333.

12. Ikeda, K., Sato, M., Tsutsumi, O., Tsuchiya, F., Tsuneizumi, M., Emi, M., Imoto, I., Inazawa, J., Muramatsu, M. and Inoue, S. 2000. Promoter analysis and chromosomal mapping of human EBAG9 gene. Biochem. Biophys. Res. Commun. 273: 654-660.

13. Iwasaki, T., Nakashima, M., Watanabe, T., Yamamoto, S., Inoue, Y., Yamanaka, H., Matsumura, A., Iuchi, K., Mori, T. and Oada, M. 2000. Expression and prognostic significance in lung cancer of human tumor-associated antigen RCAS1. Int. J. Cancer 89: 488-493.

14. Izumi, M., Nakanishi, Y., Yoshino, I., Nakashima, M., Watanabe, T. and Hara, N. 2001. Expression of tumor-associated antigen RCAS1 correlates significantly with poor prognosis in nonsmall cell lung carcinoma. Cancer 92: 446-451.

15. Kaku, T., Sonoda, K., Kamura, T., Hirakawa, T., Sakai, K., Amada, S., Ogawa, S., Kobayashi, H., Nakashima, M., Watanabe, T. and Nakano, H. 1999. The prognostic significance of tumor-associated antigen 22-1-1 expression in adenocarcinoma of the uterine cervix. Clin. Cancer. Res. 5: 14491453.

16. Kubokawa, M., Nakashima, M., Yao, T., Ito, K. I., Harada, N., Nawata, H. and Watanabe, T. 2001. Aberrant intracellular localization of RCAS1 is associated with tumor progression of gastric cancer. Int. J. Oncol. 19: 695-700.

17. Misdorp, W., Else, W., Hellmen, E. and Lipscomb, T. P. 1999. Histological classification of the mammary tumors of the dog 
and the cat. pp. 1-59. In: World Health Organization International Histological Classification of Tumors of Domestic Animals, 2nd series, vol. 7, Armed Forces Institute of Pathology, Washington.

18. Morrison, W. B. 1998. Canine and feline mammary tumors. pp. 591-598 In: Cancer in Dogs and Cats (Lippincott, W. ed.), Lippincott, Williams \& Wilkins, Philadelphia.

19. Nakakubo, Y., Hida, Y., Miyamoto, M., Hashida, H., Oshikiri, T., Kato, K., Suzuoki, M., Hiraoka, K., Ito, T., Morikawa, T., Okushiba, S., Kondo, S. and Katoh, H. 2002. The prognostic significance of RCAS1 expression in squamous cell carcinoma of the oesophagus. Cancer Lett. 177: 101-105.

20. Nakashima, M., Sonoda, K. and Watanabe, T. 1999. Inhibition of cell growth and induction of apoptotic cell death by the human tumor-associated antigen RCAS1. Nat. Med. 5: 938942.

21. Noguchi, K., Enjoji, M., Nakamuta, M., Nakashima, M., Nishi, H., Choi, I., Taguchi, K., Kotoh, K., Shimada, M., Sugimachi, K., Tsuneyoshi, M., Nawata, H. and Watanabe, T. 2001. Expression of a tumor-associated antigen RCAS 1 in hepatocellular carcinoma. Cancer Lett. 168: 197-202.

22. Oizumi, S., Yamazaki, K., Nakashima, M., Watanabe, T., Hommura, F., Ogura, S., Nishimura, M. and Dosaka-Akita, H. 2002. RCAS1 expression: a potential prognostic marker for adenocarcinomas of the lung. Oncology 62: 333-339.

23. Okamura, Y., Ma, Z., Khatlani, T. S., Okuda, M., Une, S., Nakaichi, M. and Taura, Y. 2003. Molecular Cloning of Canine RCAS1 cDNA. J. Vet. Med. Sci. 65: 913-915.

24. Owens, L. N. 1980. Classification of Tumors in Domestic Animals, 1st ed., World Health Organization, Geneva.

25. Perez, J., Day, M. J. and Mozos, E. 1998. Immunohistochemical study of the local inflammatory infiltrate in spontaneous canine transmissible venereal tumour at different stages of growth. Vet. Immunol. Immunopathol. 64: 133-147.

26. Rousseau, J., Tetu, B., Caron, D., Malenfant, P., Cattaruzzi, P., Audette, M., Doillon, C., Tremblay, J. P. and Guerette, B. 2002. RCAS1 is associated with ductal breast cancer progression. Biochem. Biophys. Res. Commun. 293: 1544-1549.

27. Rutteman, G. R., Withrow, S. J. and MacEwen, E. G. 2001. Tumor of the mammary gland. pp. 455-477. In: Small Animal Clinical Ooncology (Withrow, S. J. and MacEwen, E. G. eds.), WB Saunders Co., Philadelphia.

28. Sonoda, K., Nakashima, M., Kaku, T., Kamura, T., Nakano, H. and Watanabe, T. 1996. A novel tumor-associated antigen expressed in human uterine and ovarian carcinomas. Cancer 77: 1501-1509.

29. Sonoda, K., Kaku, T., Kamura, T., Nakashima, M., Watanabe, T. and Nakano, H. 1998. Tumor-associated antigen 22-1-1 expression in the uterine cervical squamous neoplasias. Clin. Cancer Res. 4: 1517-1520.
30. Sonoda, K., Kaku, T., Hirakawa, T., Kobayashi, H., Amada, S. Sakai, K., Nakashima, M., Watanabe, T. and Nakano, H. 2000. The clinical significance of tumor-associated antigen RCAS1 expression in the normal, hyperplastic, and malignant uterine endometrium. Gynecol. Oncol. 79: 424-429.

31. Sonoda, K., Miyamoto, S., Hirakawa, T., Kaku, T., Nakashima, M., Watanabe, T., Akazawa, K., Fujita, T. and Nakano, H. 2003. Association between RCAS1 expression and clinical outcome in uterine endometrial cancer. Br. J. Cancer 89: 546551.

32. Suzuki, T., Inoue, S., Kawabata, W., Akahira, J., Moriya, T., Tsuchiya, F., Ogawa, S., Muramatsu, M. and Sasano, H. 2001. EBAG9/RCAS1 in human breast carcinoma: a possible factor in endocrine-immune interactions. Br. J. Cancer 85: 17311737.

33. Tada, K., Oka, M., Tangoku, A., Hayashi, H., Oga, A. and Sasaki, K. Gains of 8q23-qter and 20q and loss of 11q22-qter in esophageal squamous cell carcinoma associated with lymph node metastasis. Cancer 88: 268-273.

34. Tsuchiya, F., Ikeda, K., Tsutsumi, O., Hiroi, H., Momoeda, M., Taketani, Y., Muramatsu, M. and Inoue, S. 2001. Molecular cloning and characterization of mouse EBAG9, homolog of a human cancer associated surface antigen: expression and regulation by estrogen. Biochem. Biophys. Res. Commun. 284: 210

35. Tsuneizumi, M., Emi, M., Nagai, H., Harada, H., Sakamoto, G., Kasumi, F., Inoue, S., Kazui, T. and Nakamura, Y. 2001. Overrepresentation of the EBAG9 gene at $8 \mathrm{q} 23$ associated with early-stage breast cancers. Clin. Cancer Res. 7: 3526-3532.

36. Tsuneizumi, M., Nagai, H., Harada, H., Kazui, T. and Emi, M. 2002. A highly polymorphic CA repeat marker at the EBAG9/ RCAS1 locus on $8 \mathrm{q} 23$ that detected frequent multiplication in breast cancer. Ann. Hum. Biol. 29: 457-460.

37. Underwood, J. C. E. 1974. Lymphoreticular infiltration in human tumours: prognostic and biological implications. Br. J. Cancer 30: $538-548$.

38. Walch, A. K., Zitzelsberger, H. F., Bruch, J., Keller, G., Angermeier, D., Aubele, M. M., Mueller, J., Stein, H., Braselmann, H., Siewert, J. R., Hofler, H. and Werner, M. 2000. Chromosomal imbalances in Barrett's adenocarcinoma and the metaplasia-dysplasia-carcinoma sequence. Am. J. Pathol. 156: 555566.

39. Watanabe, T., Inoue, S., Hiroi, H., Orimo, A., Kawashima, H. and Muramatsu, M. 1998. Isolation of estrogen-responsive genes with a CpG island library. Mol. Cell Biol. 18: 442-449.

40. Yang, Z. Q., Yoshida, M. A., Fukuda, Y., Kurihara, N., Nakamura, Y. and Inazawa, J. 2000. Molecular cytogenetic analysis of 17 renal cancer cell lines: increased copy number at 5q3133 in cell lines from nonpapillary carcinomas. Jpn. J. Cancer Res. 91: 156-163. 